\title{
ЭКСТРАДИСЦИПЛИНАРНОСТЬ ПОНЯТИЯ УПРАВЛЕНИЯ И ПРОЦЕССЫ ИНТЕГРАЦИИ В СОВРЕМЕННОЙ НАУКЕ
}

\section{EXTRADISCIPLINARY CONCEPT OF MANAGEMENT AND INTEGRATION PROCESSES IN MODERN SCIENCE}

M. Luskin

E. Kalinin

Summary: Analysis of the differentiation and integration of scientific knowledge as extradisciplinary scientific directions and key concepts of purpose and management shows that with a tendency towards monism, the dominant real result is the secondary pluralization of primary concepts. It is realized in a stable multiplicity of meanings of a concept that is system-forming for an ontology (expediency, control, etc.). Development leads to the emergence of new key concepts, in particular, to multi-agent, as a system of technosubjects.

Keywords: science, differentiation, integration, disciplinarity, extradisciplinarity, goal, management, manipulation, reflection.
Люскин Михаил Борисович

Дочент, Начиональный исследовательский университет «МЭИ», г. Москва

magmich@yandex.ru

Калинин Эдуард Юрьевич

старший преподаватель, Начиональный исследовательский университет «МЭИ», г. Москва

KalininEU@mpei.ru

Аннотация: Анализ дифференциации и интеграции научного знания как экстрадисциплинарных научных направлений и ключевых понятий цели и управления показывает, что при тенденции к монизму господствующим реальным результатом является вторичная плюрализация первичных понятий. Она реализуется в устойчивой множественности значений понятия, системообразующего для онтологии (целесообразности, управления и т.д.). Развитие приводит к появлению новых ключевых понятий, в частности, к мультиагентности, как системе техносубъектов.

Ключевые слова: наука, дифференциация, интеграция, дисциплинарность, экстрадисциплинарность, цель, управление, манипуляция, рефлексия.

\section{Введение}

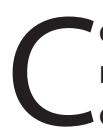
облазн телеологии, как и искушение редукционизмом - это- родственные процессы, связанные с процессами расширения пределов предметной области того или иного типа научного знания и реализации гносеологического идеала монизма. Эту тенденцию назовем, использовав неологизм монизации. Опыт изучения такой экспансии позволяет увидеть вторичный плюрализм (плюрализацию) за первичной «монизацией». Отметим две противоположные основные тенденции в первичной «монизации»:

1. сведение сложного, высоко организованного, целостного к простому и элементарному, т. е. редукция;

2. возведение простого, примитивного, элементарного к сложному, высокоразвитому, системно-организованному. Назовем эту тенденцию «экстрадукцией» или «элевацией».

В процессах дифференциации и интеграции современной науки стали образовываться понятия, направления и подходы, более общие, чем в отдельных дисциплинах. Для всех так называемых «общенаучных» понятий характерны две основные характеристики. 1. Применимость любого из них вышло за рамки отдельной науки и такое понятие приобрело общенаучный статус. 2. Каждое такое понятие (или во взаимосвязи с одним - двумя подобными) служит основой особого на- учного подхода к познанию. Эти две характеристики отличают данную группу понятий от понятий частных наук. Такие понятия по своим свойствам занимают промежуточное положение между частнонаучными понятиями и философскими категориями. Традиционное дихотомическое деление понятий науки стало в наше время недостаточным. Их качественная специфика заключается в двух свойствах:

1. они принципиально применимы во всех областях научного знания;

2. в отличие от категорий философии не обладают логико-гносеологической универсальностью. [1], [5].

Однако первый признак выделения этих «общенаучных» понятий - применимость во всех науках на самом деле ложен, вернее, излишне экстраполирован. Ни один из них не используется реально во всех науках, точнее, не используется эффективно. Например, понятие «управление», номинально используемое в физике, за исключением отдельных физических теорий, либо не применяется, либо неэффективно, либо имеет существенно иной смысл (допустим, управляющие параметры). Образованные путем не столько индуктивного обобщения эмпирических исследований, сколько метатеоретрическим синтезом тех или иных аспектов частных научных теорий эти понятия приобрели характер экстрадисциплинарных образований. Они часто связаны с существованием соответствующих подходов к познанию (системный, кибернетический, динамический, 
синергетический подходы и т. д.). Эти гносеологические и методологические феномены (понятия, подходы и дисциплины) следуют назвать экстра(над-) дисциплинарными, зафиксировав в названии их специфический характер существования и функционирования. Там, где существует метатеоретическая иерархическая онтология и плюрализм смыслов понятия, философско-методологический анализ, как правило, обнаруживает некритический и нерефлексированный монизм ученого или исследователя, связанный с их монотеоретической позицией.

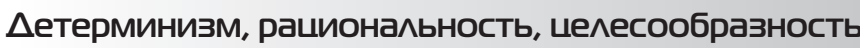

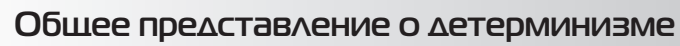

Под детерминизмом обычно понимают взаимосвязь, взаимообусловленность и взаимоопределенность вещей, процессов и явлений. Чтобы детерминизм (определенность) мог содержательно пониматься (в свою очередь быть определенным) необходимо наличие противоположного понятия, т.е. индетерминизма (неопределенности). В классической философии и в классической науке детерминизм привязан к той или иной онтологической концепции. С появлением неклассической физики соотношение определенности и неопределенности в философии науки связано не только с онтологией, но и с методологией той или иной научной теории.

При этом философская онтология детерминизма оказывается вторичной и зависит от иерархии наук (например, в виде «форм движения материи», «структурных уровней» и т. д.) и, либо эта иерархия как-то «сшивается» с помощью принципа развития материи или абсолютной идеи и т. п. при философском монизме, либо иерархия онтологий оказывается не объединенной в случае философского плюрализма. Причем в философии естествознания больше тяготеют к интеграции онтологий, а в социальной философии (исключая марксизм) - наоборот, к дифференциации [3].

\section{Прочессы Аифференшиашии и интеграшии в современной науке}

До XX в. традиционная дуальная дифференциация наук на конкретные, опытные (содержательные) и формальные (абстрактные) казалась незыблемой. Веян-в первую группу попадали естественные, затем технические и социогуманитарные науки, то во вторую группу - математика и логика. Но в XX в., особенно, начиная с его середины, доожно говорить о возникновении третьей группы наук, которая по своим характеристикам является промежуточной или синтетической. Ее можно назвать группой интегративноабстрактных наук. Это, прежде всего, системно-кибернетические дисциплины, теория колебаний и волн (и / или теория динамических систем), синергетика и компьютерные науки. В этой группе объекты каждой из наук образованы путем абстрагирования от большинства эмпирических и теоретических характеристик объектов наук всей первой группы и выделения одного или нескольких немногих свойств (колебаний и волн для ТКВ и ТДС; самоорганизации - для синергетики; системы, элементов и структуры - для теории систем, и т. д.). Одновременно с этим их степень формализации в целом выше по сравнению с большинством естественных и технических наук, но ниже, чем формализация наук второй группы. Поэтому их естественно назвать интегративно-абстрактными (-формальными) науками. Или (следуя терминологии квантовой механики, в которой гибридная модель, где часть параметров классические, а часть квантовые, называется полу (квази-) классической), назовем их полу (квази-) формальными, или полу (квази-) содержательными науками или дисциплинами [9-15].

В современном обществе возникли новые научнотехнические и социально-экономические проблемы, которые затрагивают три основные области науки: естествознание, обществознание и техникознание. Речь идет, прежде всего, о глобальных проблемах, долгосрочных социально-экономических прогнозах и комплексных научно-технических проектах. Эти интегративные процессы и одновременно эти междисциплинарные исследования можно назвать трансдисциплинарными в узком смысле слова. При этом характер интеграционных процессов имеет иную природу. Дисциплинарное знание в таких проектах выступает как ресурс, а его потребление находится вне дисциплинарных представлений. Главным средством, позволяющим реально интегрировать познавательную деятельность в этой сфере, выступает объединение математического моделирования с использованием ЭВМ, зачастую доведенное до вычислительного эксперимента. Оно применяется в исследовании сложных систем, поведение которых не удается понять на основе знания свойств их отдельно взятых компонентов. Эти новые объекты исследования могут включать в себя явления различной природы, но настолько тесно связанные, что их в принципе нельзя рассматривать независимо друг от друга. При этом теоретическое познание и практическое управление сложными системами выходит на первый план (см., например, [2]).

Особенность этого постклассического типа междисциплинарных исследований в том, что он приводит к возникновению нового типа постклассических интегральных научных дисциплин. Возникновение такого рода новых междисциплинарных направлений, увеличение их числа, значимости, их непреходящий характер в современной науке позволяют сделать вывод, что они являются новыми формами организации 
науки, которая перестраивается с изменением практических потребностей. Такие междисциплинарные направления правильнее было бы назвать над-, или транс-, или экстрадисциплинарными, имея в виду новый способ организации их идеализированной предметности.

Можно говорить о трех основных типах методологии: I) 1) Дескриптивной (описывающей) - Д; ІІ) прескриптивной (предписывающей): 2) Нормативной (Н) или оптимизирующей (целеподобной) и 3) Конструктивной (К) - целесообразной.

При этом Д-методология традиционно соотносилась с естествознанием (где описание «естественным» образом связывалось с чувственным опытом, Н-методология - с обществоэнанием, а К-методология - с технознанием. Но уже с самого начала зарождения 3-х типов методологий выяснилось, что естествознание Нового времени связано с К-методологией через эксперимент. В любой технической теории обязательно существует дескриптивный слой теоретических объектов. Обществознание связано с Д-методологией через описание ценностей, а гуманитарное знание со своим смыслом, пониманием, диалогом оказалось как-то вне такого соответствия.

Нам представляется, что переход от классической науки к постклассической знаменуется не последовательными этапами развития научных дисциплин, а взаимным проникновением альтернативных методологий и онтологий в тело каждого из типов наук. В частности для естествознания - это конструктивизация и гуманизация предмета и метода; для технознания - натурализация и гуманизация и для обществознания - это конструктивизация и натурализация.» Тогда к причинной детерминации, почти монопольно господствующей в онтологии классического естествознания, добавляется целевая детерминация (например, в форме относительности к средствам измерения и т.п.). К ценностной детерминации, преобладавшей в онтологии классического естествознания прибавляется и теснит её конструктивноцелевая. Кроме того, переход к постклассической науке сопровождается появлением нового типа интегративноабстрактных наук (например, системно-кибернетического класса). Идеализированная предметность этого типа единым образом описывает все три традиционных типа объектов: природу, общество, технику, при этом ключевой детерминацией для онтологии этого класса наук является целевая. Поэтому появляется устойчивая методологическая ориентация на то, чтобы онтологии других типов наук, как и основные типы объективной детерминации свести к одной единой целевой онтологии. Однако попытки монистического сведения разных типов детерминаций к одному (целевой и ценностной к причинной - в позитивизме; причинной и целевой - к ценностно-смысловой - в субъективизме и причинной и ценностной - к целевой - в конструктивизме) приводят к обратному итогу- к иерархии понятий, а значит и видов детерминаций внутри основного системообразующего. В частности, при стремлении единым целевым образом построить онтологию и методологию всех основных типов наук появляется иерархия, а значит и плюрализм видов целей, целевых онтологий, а, следовательно, и методологий.

\section{Методологические позичии \\ и эпистемологические гранишы преАставлений о шели и шелесообразности}

Попробуем проследить иерархичность онтологии на примере анализа основных концепций цели и управления. Как в отечественной, так и в зарубежной философии и методологии науки существуют три основные точки зрения на объективный статус понятий «цель», и «целесообразность», которые в своей совокупности и представляют собой вторичную плюрализацию целесообразности. Они альтернативны в том, насколько широко надо понимать целесообразность и применять понятие цели для характеристики того или иного класса систем.

1. Наиболее широкое понятие цели и представление о целесообразности связывается не только с существованием трех вышеназванных систем, но и существованием неживой природы. Одним из немногих представителей такой точки зрения в нашей стране является В.А. Ассеев. В его предельно широкой трактовке целеустремленность является «направленностью реакций» на поддержание и сохранение системы, которая не является исключительным свойством живых систем или систем с обратной связью, а есть выражение устойчивости систем любой природы. Понятие целесообразность в применении к неживой природе можно определить как устойчивость по отношению к внешним воздействиям. [2, с. 89-92]. Стремление динамической системы к устойчивому состоянию (аттрактору) часто в синергетике отождествляется с подобием целесообразности. Такая концепция целесообразности создает впечатление, что поведение системы действительно определено некоторым её конкретным состоянием, которое она примет в будущем и которого пока ещё нет как такового. На самом деле это не так: детерминация конечным состоянием связана с определенностью самой системы с её качеством и структурными особенностями, которые могут не существовать в текущем времени реализации того или иного режима, но существуют с самого начала в обобщенном фазовом пространстве системы. Таким образом, представления об экстремальности (оптимальности) или о об аттракторах системы как конечных состояниях недостаточны для формирования представления о целесообразности более сложных органических и, тем бо- 
лее, технических и социальных систем. Такие системы можно назвать целенаправленными.

2. Менее широкое понятие цели и представление о целесообразности связывается с существованием трех классов систем:

а) социальных,

б) биологических,

в) кибернетических.

При этом цель понимается как некоторая модель реальности, которой и должен соответствовать тот или иной процесс или явление А. Розенблют, Н. Винер и Дж. Бигелоу дают следующую классификацию поведения. Под поведением понимается любое изменение объекта по отношению к окружающей среде. Активным поведением является такое, при котором объект служит источником выходной энергии, используемой в данной специфической реакции. Целенаправленное поведение означает, что поведение допускает истолкование как направленное на достижение некоторой цели, т.е. конечного состояния, при котором объект вступает в определенную связь в пространстве или во времени с другим объектом. Телеологическое поведение - поведение с обратной связью. Обратная связь в широком смысле означает, что часть выходной энергии (выходного сигнала) некоторой системы возвращается на вход. Обратная связь в узком смысле означает, что поведение управляется с помощью величины ошибки в положении объекта по отношению к некоторой цели. Экстраполирующее (предсказывающее) поведение - поведение, где управление связано с экстраполяцией в будущее искомой цели [4, с. 285-294].

Для введения достаточно общего и продуктивного представления о целесообразности необходимо, помимо устойчивых конечных состояний, допустить оценку состояния системы на соответствие (несоответствие) данного конечного состояния, которое при наличии таких оценок и может называться целью. При этом становится очевидным, что такая оценка состояния предполагает обратную связь и управление этим соответствием, кроме того, некоторый промежуточный параметр - информацию, благодаря которой происходит сравнение и оценка. Тем самым становится ясно, что максимально общее понятие цели предполагает обратную связь, управление и информацию, т. е. класс кибернетических систем. Такие системы можно назвать целеподобными. В зависимости от разновидности второй концепции биологические и социальные системы являются либо подклассами кибернетических систем, либо независимыми классами. Если не вводится дополнительный специфический язык, специальные понятия и интерпретация этих базовых кибернетических понятий, то кибернетическая концепция целесообразности выступает как абстрактная концепция, конкретной реализацией которой может выступить тот или иной класс систем технической кибернетики. Если же специальный язык, понятия и интерпретация введены, тогда кибернетическая концепция целесообразности выступает как метатеория.

3. Максимально узкое понятие цели и представление о целесообразности связывается только с существованием человека и человечества. В такой концепции цель определяется как один из элементов поведения и сознательной деятельности человека, который характеризует предвосхищение в мышлении результата деятельности и пути его реализации с помощью определенных средств . Цель в этой концепции - идеальна независимо от того осознанна она или нет. Эта концепция в собственном смысле телеологических (целесообразных) систем. При этом деятельность понимается как специфически человеческая форма активного отношения к окружающему миру, содержание которой составляет его целесообразное изменение и преобразование в интересах людей. Она предполагает определенное противопоставление субъекта и объекта деятельности. Субъект как носитель и источник деятельности противопоставляет себе объект деятельности как материал, который, в согласии со своими собственными законами, но также и в согласии с целями человека должен получить новую форму и новые свойства превратиться из материала (предмета) в продукт (результат) деятельности.

Структура всякого элементарного акта деятельности включает в себя субъект (тот, кто действует), цель (идеальный образ результата), предмет (что преобразуется в процессе деятельности для достижения поставленной цели), средство (им преобразуется предмет), условия (с помощью происходит преобразование, но что непосредственно не участвует в этом процессе), результат и сам процесс деятельности. Целесообразный характер деятельности приводит к тому, что одним из главных её оснований является сознание, понимаемое в самом широком смысле - не только как совокупность различных внешних форм, но и как множество его внутренних результатов (потребностей, мотивов, установок, ценностей и т.д.). Сознание играет в деятельности двоякую роль: оно выступает в качестве ее внутреннего компонента, средства контроля за ходом деятельности (рефлексивное сознание) и выступает как внешняя - как источник формирования представлений о целях, смысле и оценке деятельности.

\section{Косвенное шелеполагание в социальных системах

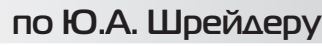

Можно выделить типы поведения, при которых стремление к некоторой цели есть наиболее эффективный способ достижения совсем иной цели. Этим обусловлено существование механизмов социального поведения с косвенным целеполаганием, когда можно 


\section{различить}

1. осознаваемую непосредственную цель поведения, к которой стремится субъект, и

2. косвенно достигаемую фактическую цель. Это могут быть и стереотипные механизмы поведения, выработанные в обществе, и механизмы внешнего управления поведением на основе косвенного целеполагания.

О поведении с косвенным целеполаганием можно говорить в том случае, если воля субъекта поведения не направлена на достижение фактической цели, которая этим субъектом может осознаваться или не осознаваться, но которая может быть выявлена исследователем. Более того, фактически достигаемая в акте поведения цель может вообще не иметь непосредственной причинной связи с непосредственной целью. В частности, можно констатировать, что более общая цель отсутствует и лишь часть поведения обусловлена необходимостью достижения конкретной цели. Остальные аспекты данного поведения - не вытекающие из его непосредственной цели - обозначим как ритуальные. В случае ритуального поведения непосредственная цель «вырождена» или автономна - она состоит в выполнении самого ритуала. Волевое усилие субъекта направлено на соблюдение формы поведения. Фактически достигаемая цель, которая придает ритуалу социальный смысл, причинно не связана с единичным актом ритуального поведения. Этот смысл возникает в результате того, ритуал становится регулярным компонентом общественного поведения. [11].

Таким образом, вместо одного понятия цели [целесообразности] мы обнаруживаем, по меньшей мере, три несводимых друг к другу концепции. Можно ли говорить о том, что смысл понятия идеальной цели включает в себя смысл понятия цели как конечного состояния, как оптимального состояния, как гомеостаза, как функционального инварианта (информационной модели) вместе с оценкой его достижения и т.д.? И да, и нет! Та или иная идеальная цель может включать в себя те или иные аспекты низших форм целесообразности, но не всегда, не все и не каждая. Здесь нет единого линейного кумулятивного процесса или процесса снятия. Итак, множественность бытия целевой детерминации приводит к объективному плюрализму и иерархии смыслов и значений понятия цели.

\section{Шелесообразность и управление Основная типология коншепший управления}

Если обратиться после целесообразности к понятию управления, то здесь также выявляется аналогичная картина. За внешним абстрактным понятием управления в кибернетике скрывается реальная множественность видов управления и соответствующих понятий, которая отнюдь не содержится в исходной абстракции.

«Если понимать человеческую деятельность как высший вид жизнедеятельности организмов, последнюю - как высший вид биологической активности и т.д., то можно мысленно построить «пирамиду», в основании которой будет понятие движения вообще и соответствующее ему предельно широкое понятие управления, понимаемое как свойство систем любой природы, обладающих минимально необходимыми для наличия этого свойства признаками, а на вершине - высший тип управления, «человеческое» управление» [3].

Самая простая иерархия понятий выглядит следующим образом:

1. равновесие, устойчивость,

2. авторегулирование,

3. управление

4. деятельность.

Кибернетизация нарушила однозначную связь понятия управления с человеческой деятельностью. Если раньше последняя была для управления ближайшим родом, то теперь эти понятия стали пересекающимися. Как следует в применении к кибернетизированному понятию «управление» трактовать смысл терминов «цель управления», «целенаправленность», «целесообразность»? Как пишет В.А. Бокарев: «если, с одной стороны, настаивать, что управление - обязательно целенаправленное, целесообразное действие, а с другой - распространять это понятие на технику и природу, то не избежать противоречия. И выходов из него три:

1. отказаться от понимания управления как сугубо целенаправленного процесса,

2. признать, что управлением «в полном смысле» является только человеческое управление,

3. попытаться изменить объем и содержание понятия «целесообразность».

Как следует характеризовать процесс управления? Именно целесообразность отличает управление от любых других воздействий и взаимодействий объектов. Признание целесообразности процесса управления важнейшей характеристикой его содержания согласуется с тем, что в качестве важнейшей характеристики системы управления была взята обратная связь. Наличие целесообразной обратной связи, таким образом, выступают как существенное в управлении, взятое в одном случае со стороны содержания, (функции), а в другом - со стороны формы (структуры) [3].

В связи с тем, что главным содержанием управления является именно его целевое содержание, рассмотрим вопрос: в чем состоят цели управления. У.Р. Эшби и Ст. Бир полагают, что основной целью является гомео- 
стазис. С одной стороны, гомеостатические процессы наблюдаются во всех системах управления. С другой стороны, в объектах, не относящихся к классу систем управления, таких процессов не наблюдается. Следовательно, гомеостазис - это качественная грань, выделяющая процесс управления среди всех других процессов материального мира.

Однако гомеостазис не охватывает собой всего существа управления. Гомеостатическая система в структурном отношении характеризуется только отрицательными обратными связями, а в функциональном постоянством цели, именно - цели самосохранения (посредством активного приспособления к среде). При усложнении среды такая система способна к развитию, но не к саморазвитию.

Гомеостазис в социальных системах проявляется иначе: человек не только сам лично приспосабливается к условиям существования, но, даже не ограничиваясь приспособлением техники к этим условиям, адаптирует саму среду к своим нуждам и целям. Еще важнее то, что выживание, самосохранение, приспособление в обществе перестают быть самоцелью, как в живой природе, хотя здесь самосохранение может реализоваться не только по отношению к особям, но и к видам. Таким образом, гомеостазис, являясь существенной чертой управления, не охватывает всей его сути. Поэтому, например, в работах В.Г. Афанасьева и И.Б. Новика управлению дается более широкая характеристика. Оно, согласно И.Б. Новику, предстает как упорядочение системы, приведение её в соответствие с определенной объективной закономерностью, действующей в данной среде. Такой подход охватывает и гомеостазис, и саморазвитие [8]. Анализ основных концепций управления и его связи с целесообразностью также выявил множественность концепций, несводимую к единому понятию и его смыслу, т.е. иерархичность и множественность концепций управления тесно связаны с аналогичными характеристиками концепции целесообразности. Однако существует всегда монистическая тенденция, которая особенно усиливается в случае математизации соответствующей области знания.

Структурная модель управления как и структурная модель деятельности ограниченно применима для социогуманитарной сферы, т.к. осознания цели отнюдь не достаточно для полной характеристики специфики индивидуального и социального управления. Цель может быть косвенной и содержаться:

1. либо в нерефлексивном сознании,

2. либо в установке в бессознательном,

3. либо как доминанта в центральной нервной системе,

4. либо природная детерминация может располагаться еще глубже.
Нас будет, прежде всего интересовать вариант, когда цель скрыта и навязывается извне. Назовем этот процесс манипуляцией.

Рассмотрим классификацию основных сфер манипулятивного воздействия. Манипуляция:

1. целями,

2. чувствами и эмоциями,

3. потребностями и интересами,

4. идеалами и ценностями,

5. символами формируется с помощью символического конструирования.

Это создание такой символической среды, в которой у объекта манипуляции формируется потребности, интересы, ценности и идеалы, приводящие к поведению, требуемому субъектом манипуляции.

Современные интегративные процессы в науке приводят не только к возникновению новых дисциплин, но и к развитию таких экстрадисциплинарных научных направлений как кибернетика. В частности, ряд исследователей говорит о поствинеровской (постклассической) кибернетики, одним из ключевых понятий которой является мультиагентность. Мультиагентная (или многоагентная) система состоит из множества взаимодействующих интеллектуальных автономных агентов и пассивной среды, в которой агенты существуют и на которую могут влиять. Ключевой элемент подобных систем -агент, под ним в общем случае понимается любая сущность, «которая находится в некоторой среде, воспринимает ее посредством сенсоров, получая данные, которые отражают события, происходящие в среде, интерпретирует эти данные и действует на среду посредством эффекторов».

Агентам присущи следующие свойства: автономность; коммуникативность (взаимодействуют с другими агентами); целенаправленность и активность (наличие цели и способность выполнять определенные действия для ее достижения); ограниченность представления (ни у одного из агентов нет представления обо всей системе в целом, как нет и агента, управляющего действиями других агентов: т. е. мультиагентные системы - децентрализованные системы, которым присуща самоорганизация. [6]

\section{ЗакАючение}

Анализ процессов дифференциации и интеграции научного знания на примере понятий цели и управления показывает, что при сохраняющейся тенденции к монизму господствующим реальным результатом является вторичная плюрализация первичных понятий. Она реализуется в устойчивой множественности значений понятия, системообразующего для того или иного типа онтологии (целесообразности, управления и т.д.). 
ЛИТЕРАТУРА

1. Алешин А.И. 06 одном подходе к анализу философских категорий // Проблемы методологии философского исследования. - Горький: ГГУ, 1972.

2. Ассеев В.А. Экстремальные принципы в естествознании. - Я.: ЛГУ, 1977.

3. Бокарев В.А. Человеческая деятельность и диалектика развития понятия «управление» // Кибернетика и диалектика. - М.: Наука, 1984. - С. $39-45$.

4. Винер Н. Кибернетика. - М.: Советское радио, 1968.

5. Готт В.С., Урсул А.Д., Семенюк Э.П. Категории современной науки. - М.: Мысль, 1985.

6. Лихтенштейн В.Е. и др. Мультиагентные системы: самоорганизация и развитие. М.: Финансы и статистика, 2018. 264c

7. Неймарк Ю.И. Динамические системы и управляемые процессы М., Наука 1984

8. Новик И.Б. Кибернетика М. 1963

9. Психологические механизмы регуляции социального поведения. - М.: Наука, 1979.

10. Садовский В.Н Основания общей теории систем, м.: Наука, 1974.

11. Системные исследования, ежегодники. »1969-1981. м.: Наука.

12. Эшби Р. Введение в кибернетику М. 1959

○ Люскин Михаил Борисович (magmich@yandex.ru), Калинин Эдуард Юрьевич (KalininEU@mpei.ru).

Журнал «Современная наука: актуальные проблемы теории и практики»

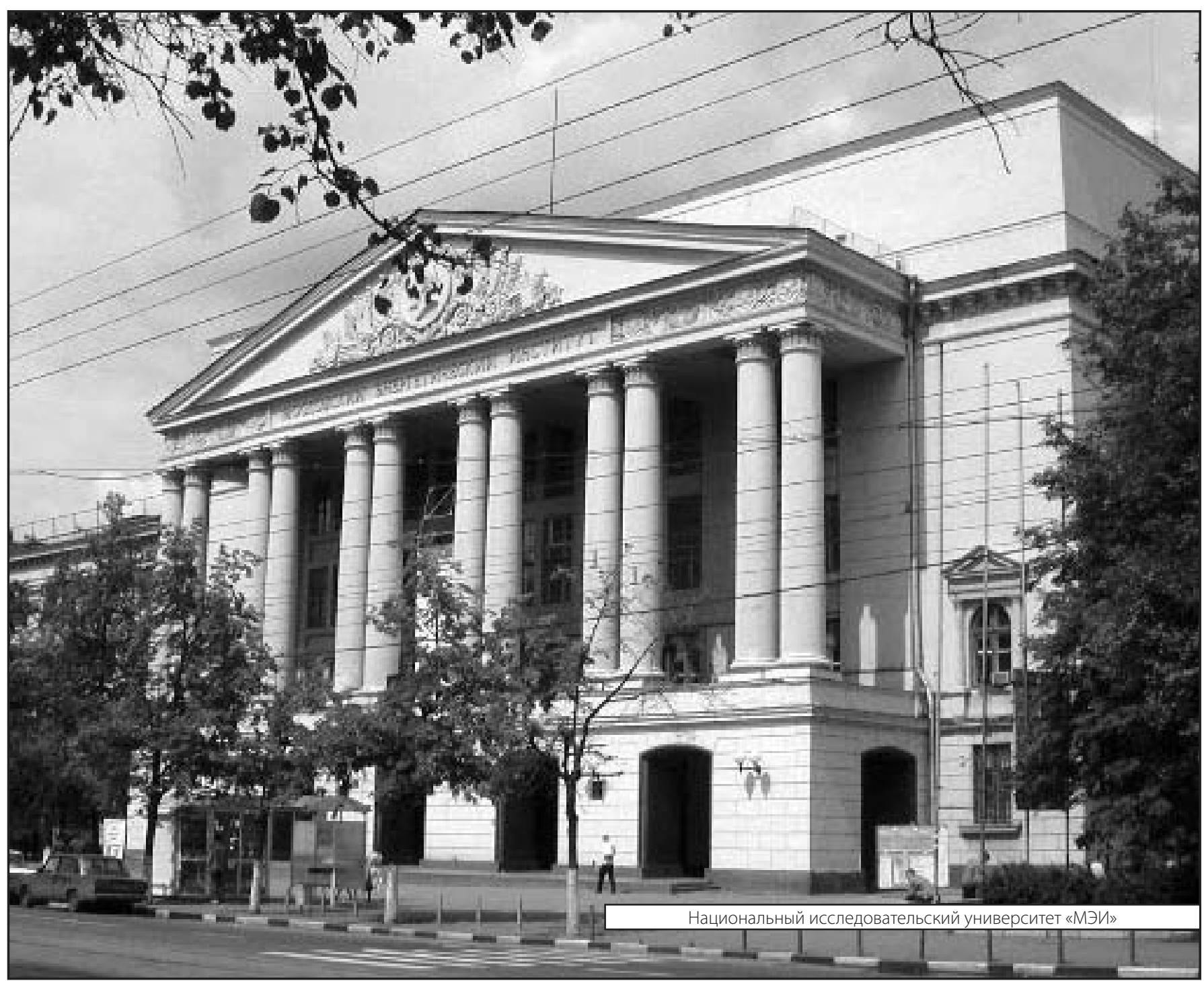

